\title{
Research on Consumer Behavior in Mobile Internet Environment Junli Zhang ${ }^{1, \text { a }}$ \\ ${ }^{1}$ Xijing University, Xi'an, Shaanxi, 710123, China
}

\section{Keywords: Consumer Behavior, Mobile Internet Environment}

\begin{abstract}
In recent years, with the rapid development of mobile commerce at home and abroad, users' consumption through the mobile terminal network increased. In order to realize the continuous development and innovation of the mobile electronic business model and e-commerce application model, it is very important to study the behavior of consumers under the background of mobile network. Therefore, this paper will study the behavior of consumers in the mobile Internet environment with a view for the relevant researchers to learn from it.
\end{abstract}

\section{Introduction}

In recent years, China and foreign mobile business has shown a rapid development trend, with mobile phones, handheld computers and other terminals for the rapid increase in wireless Internet access users. Mobile commerce applications are also widely used, such as mobile payment, mobile shopping, mobile entertainment, mobile booking, mobile lottery, etc., with the popularity of 3G applications, mobile business applications will continue to innovate. In order to promote the mobile e-commerce application model, business model innovation, for the mobile Internet environment and consumer behavior-related research is very important. Here will be in the mobile Internet environment, consumer behavior, decision-making process, consumer behavior factors, consumer behavior model and other issues discussed. In order to better study the consumer behavior in the mobile Internet environment, we first discuss the traditional market environment and the traditional Internet environment, consumer behavior, and then by comparing the two environments of consumer behavior characteristics, model to build mobile Internet consumer behavior model in the environment, consumer behavior model.

\section{The Consumer Behavior in Traditional Market Environment}

Consumption is a kind of economic behavior that human beings satisfy their own desires through consumer goods, including the causes of consumers' consumption demand, the way consumers meet their own consumption needs, and the factors that affect consumer choice. Consumption is divided into productive consumption and productive consumption, rather than productive consumption can be divided into consumption and other non-production areas of consumption; consumer behavior in the study of consumption is generally refers to the consumption of life.

Consumers have a narrow and broad understanding. Narrow understanding refers to the purchase and use of various consumer goods or services of individuals and families, broad sense refers to the purchase, use of various products and services of individuals or organizations. Here mainly study the narrow angle of the consumer.

Consumer behavior also has a narrow and broad understanding. Narrowly speaking it only refers to the consumer's purchase behavior and the actual consumption of consumer information; broadly speaking, refers to the consumer to obtain, use, disposition of consumer goods taken by the various actions and before and decided to determine the action process, and even including the acquisition of consumer income and a series of complex processes. Consumer behavior in traditional market environment is influenced by the psychological characteristics of consumers (including personality preferences, motivations and emotions, etc.) and the external environment (including social culture, marketing activities, etc.).

In academia, many scholars have endeavored to explore the rules of consumer buying in traditional market environments, such as customary theory, information processing theory, risk 
reduction theory, marginal utility theory and other consumer buying behavior theory, these theories can be used Explain the consumer's buying behavior. Scholars have also done a lot of research on the consumer behavior model and put forward some representative of the typical model, such as: consumer buying behavior of the general model; Kotler stimulus response model; Nicosia mode. These patterns can explain the causal relationship between all or local variables in the consumer buying behavior from a certain point of view. And enterprises in the marketing process to follow the basic laws of consumer behavior: AIDMA, that is, with advertising messages to attract consumers to pay attention to consumers began to be interested, to the consumer demand and desire, and potentially in the head to retain memory, and finally produce a buying action.

Because consumers want to buy the type of goods, prices, personal ability and economic conditions and other factors, consumers purchase decision-making process is sometimes relatively simple, sometimes more complex. But in general, the consumer purchase decision-making process includes five links: problem awareness, information collection, evaluation options, purchase and post-purchase evaluation.

\section{The Consumer Behavior in Mobile Internet Environment}

As the Internet tariffs continue to decline, the popularity of smart phones, mobile phone users living habits change, so that the mobile Internet environment, the rapid development of online shopping momentum, mobile Internet environment, consumer behavior mainly presents the following characteristics: (1) Anytime, anywhere. Compared to the traditional online shopping, mobile network environment shopping is very casual, shopping almost free from time, space constraints. As long as the mobile terminal in the hands, consumers can anytime, anywhere through the mobile terminal Internet browsing, parity, order to complete the purchase. (2) More personalized. As mobile terminals (such as mobile phones) are more private than the fixed computer, each mobile terminal has demonstrated the owner's personality. With the bar code, two-dimensional code scanning, graphics and voice search and other human-computer interaction technology mature, mobile e-commerce can make a clear intention of the user faster and more directly to find the target goods. (3) More integrity. Because the mobile phone (such as mobile phone) number is unique, the user information stored on the mobile phone SIM card can determine the identity of a user, which has the basis of credit certification, so the use of mobile phone shopping than consumers in the fixed network Users will pay more attention to their credibility, virtually more to promote their more honest.

There are no significant differences in the way consumer consumption behavior in the mobile Internet context compared with the consumer behavior in the traditional network background. However, due to the strong personal characteristics of the mobile network receiving terminal, the consumption in the mobile network environment the consumer behavior model of the individual characteristics of strong, mobile Internet background consumer behavior is the main feature of personalized. Personalized composition includes: personal information in the demographic category, personal preferences, environmental information and merchandise content information. Personalization mainly includes the following two points: First, in terms of consumers, the design of personalized to help consumers effectively access the relevant services or products; Second, the business, the design of personalized help to help businesses Limit the scope of potential consumers to meet the individual needs of consumers and product demand, thereby enhancing their own sales situation. In essence, personalization is based on consumer preferences and characteristics, combined with environmental factors, carried out by businesses and consumer matching processes. Mobile personalized service refers to the goods or service providers based on the potential consumer's identity, age, personal preferences and job characteristics and other factors, to provide consumers with targeted personalized service needs. Suppliers can provide a convenient personalized service directly to the consumer population. The crowd resonates with the personality of goods, and according to the customization of goods or homemade to reflect their own personalized requirements, and this personalized service is the most critical part of how the most favorable time, the most appropriate information through the most appropriate The way to the most 
suitable person.

Although the mobile Internet and the traditional Internet network environment is not the same, but the nature of the network interconnection is the same, so in the mobile Internet environment consumption and traditional Internet environment, the consumption process is roughly the same, are to experience attention, interest, search, selection, action, sharing and other steps. In the mobile Internet environment, due to the terminal capacity, bandwidth and other applications, coupled with the characteristics of consumer behavior debris time, so people in the mobile Internet, but also in the mobile Internet environment, the time to search for information will be limited, the information collection may not be sufficient, in this session, there will be differences between the two, but in the mobile state of the consumer through the mobile terminal and friends one-on-one exchange of information, To a degree to make up for the mobile Internet environment, inadequate information collection. As the time for mobile e-commerce to obtain information is short, the decision-making time is shortened, so the decision-making process becomes real-time and short-lived, the decision becomes complicated, the game is inevitable. Game is divided into complete, incomplete information game, plus static and dynamic combination of two, in business activities due to incomplete information and action must exist in the relationship, incomplete information static and dynamic game is the most common and most realistic of the two games. In the incomplete information dynamic game, the participant's behavior has the function of passing the information, the game participant constantly through the current thing, constantly observes the opponent to make the choice to verify the previous hypothesis, and then through this posterior probability to correct the assumptions made and gets the right judgment about the opponent right.

Consumers in the context of mobile Internet consumer impact factors similar to the traditional environment, not only consumption drivers, consumer attitudes and consumer attitudes and other factors, including economic conditions, social class and group guidance and other factors, which is the traditional network background consumer spending the impact factor is very similar. However, in the mobile network terminal use, consumers can be based on their own preferences anytime, anywhere network access, mobile networks and mobile network terminals with the characteristics of private, so consumer behavior in addition to the traditional network environment and traditional market factors of the restrictions, but also to a large extent by their own personality characteristics, the ability to receive mobile terminals, terminal use environment and regional consumer culture atmosphere.

First of All, Their Own Personality Characteristics. Orport once said: People's individual behavior is very different and these differences also have stability across time and space. Consumer behavior has a very prominent impulse and urgency, therefore, consumers of their own personality characteristics will make their consumption behavior there is a big difference.

Second, Mobile Terminal Capabilities. Compared with the traditional network of computer use, mobile network background, the consumer is mainly through the mobile phone network access, and mobile phones in the consumer when faced with the biggest problem is the scope of the restrictions, that is, the screen is small, Especially for those who are not 3G mobile phone, although the performance of $3 \mathrm{G}$ mobile phone has been greatly improved, but compared with the ordinary computer, its product display function is still relatively weak. It is because consumers spend time. Coupled with the lack of mobile device display capabilities, and mobile network background communication difficulties, making the mobile Internet environment, consumer spending decisions made is more difficult.

Again, the Terminal Using Environment. Mobile network and traditional Internet in the technical support on a certain difference, mainly for the mobile network transmission is relatively small, the mobile terminal's receiving capacity is relatively weak and the use of the agreement is different. The traditional network signal transmission is relatively stable, the signal is generally not exhausted, it will not be interrupted, and mobile network transmission is often affected by the construction, climate and other interference effects of signal transmission instability, and even signal interruption; signal transmission in a certain insecurity, it is easy due to interference and damage to others were intercepted the problem. It is because of the interference and impact of these 
factors, businesses in the mobile platform display, had to consider the actual situation of consumer network delivery, which seriously restricts the purchase behavior of consumers.

Finally, the Regional Consumer Culture Environment. Private service terminals, instant service requirements, mobility of service objects and convenience of service is the characteristics of mobile e-commerce. The future development of mobile e-commerce needs to provide people with regional, community and mobile business services centered on the needs of the public. This is due to its salient features and a region of mobile network culture atmosphere on the consumer's mobile network consumption will have a very important impact. Regional mobile network consumer culture prevails, the consumer mobile network consumption will be more likely and the transaction will be more at ease.

\section{Conclusion}

Through the above comparison of consumer behavior in different market environment, we find that, although there are some differences in consumer behavior, but the nature of consumer behavior will not change, but the carrier of consumer behavior will change. So consumer behavior is bound to be affected by these carriers, the impact of different carriers on consumer behavior is of course not only the same. Consumer behavior in a mobile Internet environment is determined by the nature of mobile technology and mobile devices in a mobile business environment.

\section{References}

[1] Wang Huinong. Western scholars on consumer buying behavior of the five models[J]. Foreign social science $.1993,05$.

[2] Liao Wehong. Consumer Internet environment, consumer behavior research [J]. Science and Technology Management Research .2013, 10: 179-183.

[3] Yang Xiyong. Analysis of consumer purchasing decision-making process[J]. Finance and Trade Research. 1990 (5): 35-37.

[4] Xue Huo Na. Internet under the influence of consumer behavior and corporate marketing strategy [J]. Journal of Xi'an Engineering Science and Technology .2006, 4: 235-238.

[5] Jia Yuemei. Internet age consumer behavior characteristics and marketing strategies[J]. Modern Finance .2001 (8): 53-55. 